POS PROCEEDINGS

\title{
Model comparison and experimental constraints
}

\section{Davide Meloni*}

Dipartimento di Matematica e Fisica, via della Vasca Navale 84, 00146 Rome (Italy)

E-mail: meloniefis.uniroma3.it

In this talk I consider two different relations between the leptonic mixing angles, arising from models based on $S_{4}$ and $A_{4}$, and study whether they can be distinguished at superbeam facilities, namely T2K, NOvA and T2HK.

16th International Workshop on Neutrino Factories and Future Neutrino Beam Facilities - NUFACT2014, 25 -30 August, 2014

University of Glasgow, United Kingdom

${ }^{*}$ Speaker. 


\section{Introduction}

The recent measurement of a non-vanishing $\theta_{13}$ by Daya Bay [1] and RENO [2] has exerted some pressure on models for neutrino mixing based on the permutation groups (like $A_{4}$ and $S_{4}$, [3]), as they are generically constructed to give at leading order very specific patterns in which $\theta_{13}=0$. Corrections from the charged sector or next-to-leading contributions to the neutrino mass matrix have to be invoked to correct such patterns and make the models compatible with the experimental data. The usual approach to model building is that of considering a Lagrangian invariant under a flavour group $G$ and to subsequently break $G$ into two different subgroups in the charged lepton and neutrino sector. The structure of $G$ can also be reconstructed from the residual symmetries of the mass matrices after symmetry breaking. The authors of [4] assumed that the residual symmetries in both the charged lepton and neutrino sectors are one-generator groups. Indicating with $S_{i}$ and $T_{\alpha}(\alpha=e, \mu, \tau)$ the generators of the $Z_{2}$ and $Z_{m}$ discrete symmetries of the neutrino and charged leptons mass matrices, the previous condition implies that $\left\{S_{i}, T_{\alpha}\right\}$ form a set of generators for the flavor group $G$ for given $i$ and $\alpha$, with the meaning that all other symmetries appear accidentally. The structure of the generators is restricted by the additional requirements to be elements of $S U(3)$, for which $\operatorname{Det}\left[S_{i}\right]=\operatorname{Det}\left[T_{\alpha}\right]=1$, so they can be written as:

$$
\begin{aligned}
& S_{1}=\operatorname{diag}(1,-1,-1), \quad S_{2}=\operatorname{diag}(-1,1,-1), \quad S_{3}=\operatorname{diag}(-1,-1,1) \\
& T_{e}=\operatorname{diag}\left(1, e^{2 \pi i k / m}, e^{-2 \pi i k / m}\right), \quad T_{\mu}=\operatorname{diag}\left(e^{2 \pi i k / m}, 1, e^{-2 \pi i k / m}\right), \\
& T_{\tau}=\operatorname{diag}\left(e^{2 \pi i k / m}, e^{-2 \pi i k / m}, 1\right) .
\end{aligned}
$$

The definition of $G$ requires a relation linking $S_{i}$ and $T_{\alpha}$, assumed to be:

$$
\left(S_{i} T_{\alpha}\right)^{p}=\left(U_{P M N S} S_{i} U_{P M N S}^{\dagger} T_{\alpha}\right)^{p}=I .
$$

The lack of additional symmetry in $G$ has the direct consequence that the mixing angles are not all fixed but rather present some interesting correlations that we want to test at neutrino facilities. In this respect, we have selected two models from [4], called $1 T$ and $2 T$, which give rise to different correlations and have been shown to be compatible with the current experimental data in the neutrino sector. The expected event rates implied by $1 T$ and $2 T$ are analyzed at T2K, NOvA and T2HK, with the aim of identifying the regions in the $\left(\theta_{13}, \delta\right)$-plane where the models can be distinguished at some confidence level. It is important to stress that such correlations are leading order predictions and do not take into account possible higher order effects into the lepton mass matrices of new-physics effects [5].

In our numerical computations we consider the mixing angles to vary within the $2 \sigma$ intervals taken from [7]:

$$
\begin{aligned}
\sin ^{2} \theta_{23} & =0.386_{-0.038}^{+0.062} \\
\sin ^{2} \theta_{13} & =0.0241_{-0.0048}^{+0.0049} \\
\sin ^{2} \theta_{12} & =0.307_{-0.032}^{+0.035},
\end{aligned}
$$

whereas the $\mathrm{CP}$ phase is left free to vary in the whole $[0,2 \pi)$ range. We consider the mass differences as constant quantities, $\Delta m_{31}^{2}=2.4 \times 10^{-3} \mathrm{eV}^{2}, \Delta m_{21}^{2}=7.5 \times 10^{-5} \mathrm{eV}^{2}$, since the models studied in this paper do not give any information on the neutrino masses. 


\section{A summary of the models $1 T$ and $2 T$}

The two models under consideration both have $T_{\alpha}=T_{e}$. The first model, called $1 T$, uses the generator $S_{1}=\operatorname{Diag}(1,-1,-1)$ and the pair of values $(p, m)=(4,3)$, which corresponds to the group $S_{4}$ (see, i.e., [6]). The obtained relations among the mixing angles are:

$$
\cos ^{2} \theta_{12}=\frac{2}{3 \cos ^{2} \theta_{13}}
$$

and

$$
\tan 2 \theta_{23}=-\frac{1-5 s_{13}^{2}}{2 \cos \delta s_{13} \sqrt{2\left(1-3 s_{13}^{2}\right)}} .
$$

For the $2 T$ model, which uses the generator $S_{2}=\operatorname{Diag}(-1,1,-1)$ and the pair of values $(p, m)=(3,3)$, which corresponds to the group $A_{4}$, we get:

$$
\sin ^{2} \theta_{12}=\frac{1}{3 \cos ^{2} \theta_{13}}
$$

and

$$
\tan 2 \theta_{23}=\frac{1-2 s_{13}^{2}}{\cos \delta s_{13} \sqrt{2-3 s_{13}^{2}}} .
$$

In Fig.(1) we clearly see that the resulting parameter space in the $\left(\theta_{13}, \delta\right)$-plane spanned by the two models is quite different. The allowed regions of the atmospheric and solar angles are instead

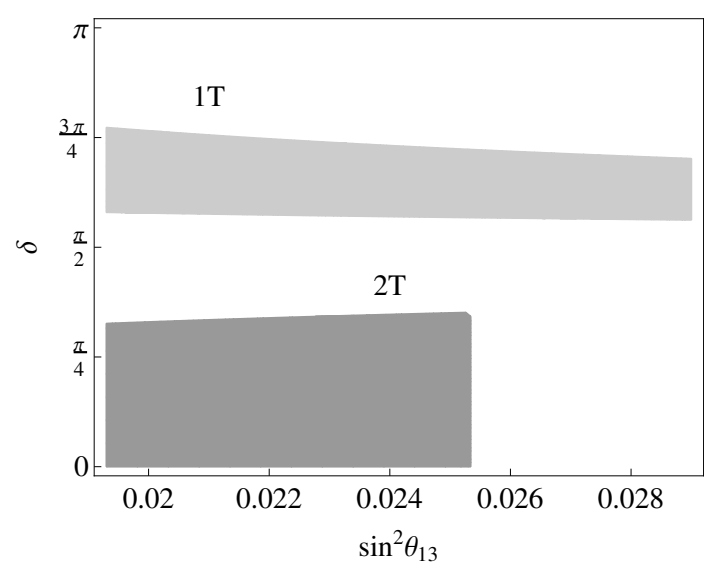

Figure 1: Allowed values of $\delta$ as a function of $\sin ^{2} \theta_{13}$ as derived imposing the correlations among the mixing parameters for the model $1 T$ and $2 T$.

summarized in Fig.(2). As we can see, two very distinct intervals for the solar angles are implied by the two models, so a strong improvement in the measurement of the solar angle could be enough to distinguish among $1 T$ and $2 T$. On the other hand, there is a large overlap in the allowed $\sin ^{2} \theta_{23}$, due to the still relatively large uncertainty affecting the determination of this angle. 




Figure 2: Allowed ranges for $\sin ^{2} \theta_{12}$ and $\sin ^{2} \theta_{23}$ for models $1 T$ (light gray) and $2 T$ (dark gray).

\section{Models at long baseline neutrino experiments}

In order to assess the capabilities of a given facility to tell $1 T$ from $2 T$ we adopt the following strategy:

- for any pair of the mixing parameters $\left(\bar{\theta}_{13}, \bar{\delta}\right)$ in the regions allowed for the model $2 T$ we compute the expected number of events $N_{\alpha, i}^{2 T}\left(\bar{\theta}_{13}, \bar{\delta}\right)$ for a given final flavour $\alpha$ and neutrino energy bin $i$ ( $\theta_{12}$ and $\theta_{23}$ are then determined from eqs.(2.3)-(2.4));

- we then compare $N_{\alpha, i}^{2 T}\left(\bar{\theta}_{13}, \bar{\delta}\right)$ to $N_{\alpha, i}^{1 T}\left(\theta_{13}, \delta\right)$, where now the mixing parameters are those of the competing model $1 T$. In this procedure, we are implicitly assuming that the model $2 T$ and the pair $\left(\bar{\theta}_{13}, \bar{\delta}\right)$ are the one chosen by Nature;

- in the next step, we minimize the following $\chi^{2}$ variable over $\theta_{13}$ and $\delta$ in the $1 T$ allowed parameter space [8]:

$$
\chi^{2}=\Sigma_{\alpha, i} \frac{\left[N_{\alpha, i}^{1 T}\left(\theta_{13}, \delta\right)-N_{\alpha, i}^{2 T}\left(\bar{\theta}_{13}, \bar{\delta}\right)\right]^{2}}{\sigma_{\alpha, i}^{2}},
$$

where the uncertainty is given by:

$$
\sigma_{\alpha, i}^{2}=N_{\alpha, i}^{2 T}\left(\bar{\theta}_{13}, \bar{\delta}\right)+B_{\alpha, i}+\left(n_{\alpha} N_{\alpha, i}^{2 T}\left(\bar{\theta}_{13}, \bar{\delta}\right)\right)^{2}+\left(b_{\alpha} B_{\alpha, i}\right)^{2},
$$

in which $B_{\alpha, i}$ is the background associated to $N_{\alpha, i}^{2 T}\left(\bar{\theta}_{13}, \bar{\delta}\right), n_{\alpha}$ the overall systematic error related to the determination of $N_{\alpha(\beta), i}$ and $b_{\alpha}$ that of $B_{\alpha, i}$. For the sake of simplicity, $n_{\alpha}$ and $b_{\alpha}$ are constant in the whole energy range;

- if the obtained minimum is larger than some reference $\chi^{2}$ value, $\chi_{\min }^{2} \geq \chi_{\text {cut }}^{2}$, then in the point $\left(\bar{\theta}_{13}, \bar{\delta}\right)$ the two models can be distinguished at a given confidence level. The ensemble of such points identifies the wanted regions. 
Obviously, the procedure can also be applied in the reverse order, that is considering $1 T$ as the true model and finding a minimum of the $\chi^{2}$ function in the $2 T$ parameter space. The results will then be presented in the $1 T\left(\theta_{13}, \delta\right)$-plane.

In the following numerical simulations [9], we will proof our strategy at three different experimental setups: NOvA, T2K and T2HK. All events rates are computed using exact numerical probabilities in matter.

\section{Results from NO $v \mathrm{~A} \oplus \mathrm{T} 2 \mathrm{~K}$ and $\mathrm{T} 2 \mathrm{HK}$}

In this section, we briefly consider the experimental setups used in our numerical simulations.

- the NOvA detector [10] is a $14 \mathrm{kt}$ totally active scintillator detector (TASD), located at a distance of $810 \mathrm{~km}$ from Fermilab, with an off-axis angle of $0.8^{\circ}$ from the NuMI beam. Our simulation is mainly based on the files provided by the GLoBES software [11,12], with migration matrices from [13] and kindly provided by one of the authors of [14]. In this way, the signal and backgrounds events released by the NOvA Collaboration are reproduced [10]. For the sake of simplicity, we take all systematics effects at the level of $5 \%$, that is $n_{\alpha}=b_{\alpha}=0.05$ for $\alpha=e^{-}, e^{+}, \mu^{-}, \mu^{+}$.

- for the T2K we consider the Super-Kamiokande water Cerenkov detector of fiducial mass of $22.5 \mathrm{kt}$, placed at a distance of $295 \mathrm{~km}$ from the source beam from J-PARC, at an offaxis angle of $2.5^{\circ}$. Our numerical simulation have been performed based to the information provided in the corresponding GLoBES files, described in [12], to which we refer for details.

- for the T2HK setup we follow the proposal and the Letter of Intent presented in [15], with a WC detector with a fiducial mass of $560 \mathrm{kton}$, placed at a distance of $295 \mathrm{~km}$ from the source. We assume again $n_{\alpha}=b_{\alpha}=0.05$.

Taken individually, NOvA and $\mathrm{T} 2 \mathrm{~K}$ have the potential to make some sort of discrimination among the $1 T$ and $2 T$ models, depending on the assumed values of $n_{\alpha}$. In particular, we have found that no distinction is possible if we assume that $2 T$ is the correct model, for any value of $n_{\alpha}$. On the other hand, under the assumption that $1 T$ gives the values of the mixing parameters chosen by Nature and $n_{\alpha}=0.05$, a limited discrimination is possible for those points in the $\left(\theta_{13}, \delta\right)$-plane with the largest possible values of the CP-phase, as it can be seen in Fig.(3). In both plots, the points above the solid lines, $\delta \gtrsim 2.06$, identify the region where the two models can be distinguished at the $90 \%$ of confidence level, using both appearance and disappearance channels. As expected, the capability of the considered facilities to distinguish the two models is almost independent on the value of $\theta_{13}$, given its small variation range. For values of $n_{\alpha}$ as large as $10 \%$ no distinction is possible. The sensitivities are the results of a strong synergy among the appearance and disappearance channels; in fact, we have observed that:

- the appearance channel alone cannot give any useful information;

- the $v_{\mu} \rightarrow v_{\mu}$ transition alone does not allow any discrimination among $1 T$ and $2 T$. However, when used in combination with the $v_{\mu} \rightarrow v_{e}$ channel, the disappearance transition sorts some 



Figure 3: Regions in the $1 T$ parameter space where the $1 T$ and $2 T$ models can be distinguished at $90 \%$ confidence level, using the appearance and disappearance channels. Left plot: for the NOvA setup. Right plot: for the T2K setup.

effects, due to the ability of measuring $\theta_{23}$ whose allowed ranges are slightly different in the two models.

A different situation arises if we combine the simulated data from both experiments. The most interesting feature is that a (reduced) region in the $2 T$ parameter space appears where the two models can be distinguished. It involves values of $\delta$ no larger than 0.2 , and only for values of
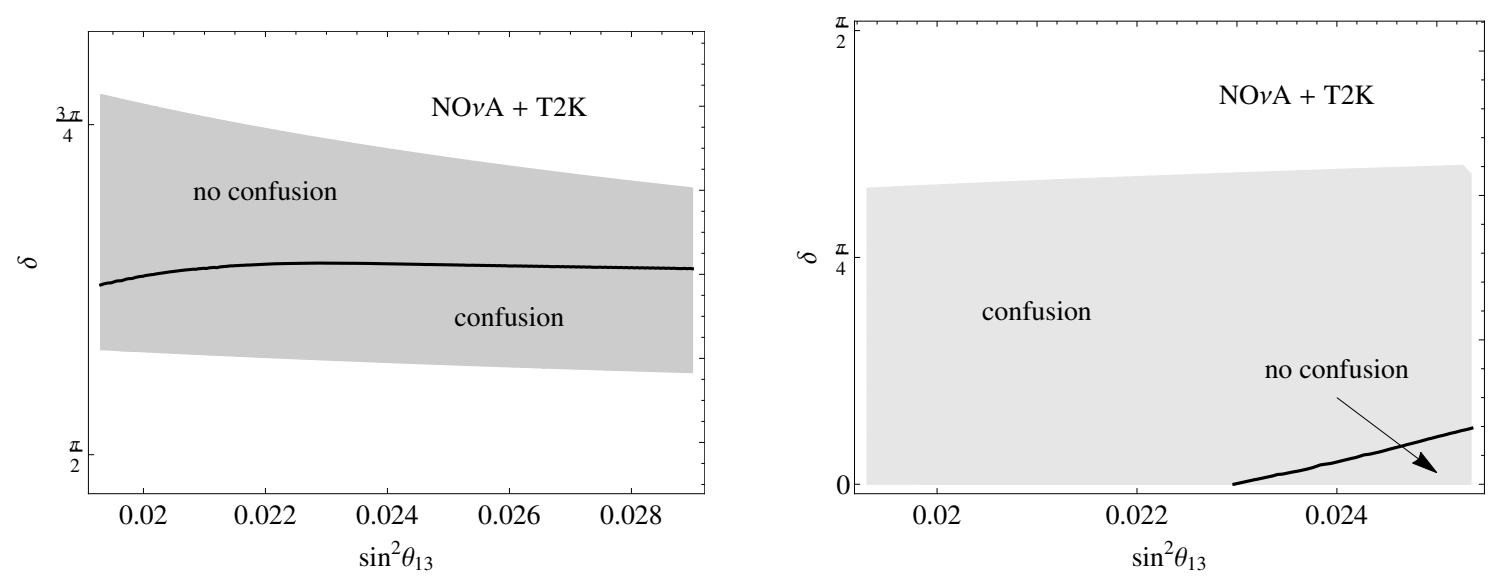

Figure 4: Regions in the $1 T$ parameter space (left panel) and $2 T$ parameter space (right panel) where the two models under investigation can be distinguished at $90 \%$ confidence level, combining the results from both NOvA and T2K.

the reactor angle close to its upper bound. In the $1 T$ parameter space, we observe only a modest improvement with respect to the case of Fig.3, due to the fact that the $\chi^{2}$ functions of the two setups are very similar in the portion of the parameter space considered, so that no powerful synergy is at work when combining the data. The different sensitivities observed in the $1 T$ and $2 T\left(\theta_{13}, \delta\right)$-plane 
are easily understood in terms of intrinsic clones [16], that is in terms of points in the parameter space with the same number of expected events.

For the T2HK setup, we get a much better capability of distinguishing the models, Fig.(5); in fact, in both $1 T$ and $2 T$ parameter spaces the regions where confusion is possible (at $99 \%$ and 99.9\% CL) are confined into thin stripes close to the lower (1T) and upper (2T) bounds, thus making this facility quite appropriate for model selection. The good performance with respect to the T2K setup has to be ascribed to the interplay between a larger detector mass and the use of the antineutrino modes. In particular, we have verified that the inclusion of the antineutrino mode into the analysis is crucial to get the sensitivities shown in Fig.(5) which, otherwise, would be a rescaled version of the T2K results shown in the right panel of Fig.(3).
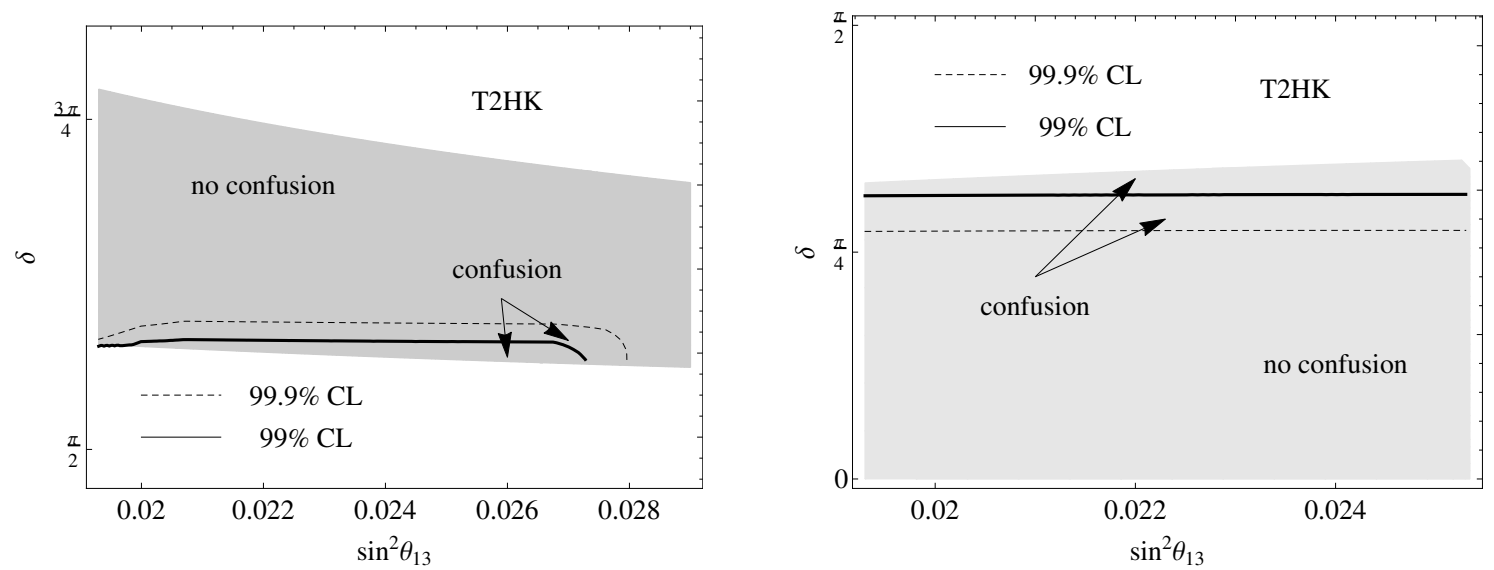

Figure 5: Regions in the $1 T$ parameter space (left panel) and $2 T$ parameter space (right panel) where the two models under investigation can be distinguished at 99\% confidence level (solid line) and 99.9\% confidence level (dashed line), in the case of the T2HK experimental setup.

A summary of the previous considerations is presented in Tab. 1 where, for each of the facilities and combination analyzed above, we reported our estimates of the range of values of the CP phase where distinction is possible among the $1 T$ and $2 T$ models. Since these ranges are modulated by $\theta_{13}$, we use "upper bound" to indicate the upper border of the $1 T$ allowed parameter space.

\begin{tabular}{|c||c|c|c|c|}
\hline \hline \multicolumn{4}{|c|}{ Approximate ranges in $\delta$} \\
\hline \hline & NOvA & T2K & NOv A + T2K & T2HK (99\% CL) \\
\hline $1 T$ & {$[2.06$, upper bound $]$} & {$[2.06$, upper bound $]$} & {$[2$, upper bound $]$} & {$[1.83$, upper bound $]$} \\
\hline $2 T$ & - & - & {$[0,0.1]$ for large $\theta_{13}$} & {$[0,1]$} \\
\hline \hline
\end{tabular}

Table 1: Estimates of range of values of $\delta$ where distinction is possible among the $1 T$ and $2 T$ models for the facilities analyzed in this paper. "Upper bound" refers the upped border of the allowed region for the $1 T$ model. Dashes indicate that no discrimination is possible.

\section{Conclusions}

Starting from two different neutrino mixing sum rules we have studied if, and to which extent, 
NOvA, T2K and T2HK are able to falsify one of them in favor of the other. This is due to the fact that the two sum rules identify different set of values of the neutrino mixing parameters, namely different regions in the CP phase $\delta$ and $\theta_{12}$ and partially overlapping regions for $\theta_{13}$ and $\theta_{23}$, all of them compatible with the experimental values at $2 \sigma$. Our numerical simulations have shown that NOvA and T2K taken alone have the capabilities to tell the $1 T$ model from the $2 T$ model at $90 \%$ of confidence level, reducing the portion in the $\left(\theta_{13}, \delta\right)$-plane of the $1 T$ model where confusion is possible. In the $2 T$ parameter space we revealed a much worse performance, unless the combination of NOvA $+\mathrm{T} 2 \mathrm{~K}$ data is taken into account, and only in a very limited region at large $\theta_{13}$ and small $\delta$. On the other hand, the T2HK experimental facility, taking full advantage of a larger detector mass and of the use of the $\bar{v}_{\mu}$ flux compared to the T2K setup, has a much better performance in terms of model selection in both parameter spaces, leaving aside only a small portion of values of $\delta$ where confusion is still possible.

\section{References}

[1] F. P. An et al. [DAYA-BAY Collaboration], Phys. Rev. Lett. 108, 171803 (2012) [arXiv:1203.1669 [hep-ex]].

[2] J. K. Ahn et al. [RENO Collaboration], Phys. Rev. Lett. 108 (2012) 191802 [arXiv:1204.0626 [hep-ex]].

[3] G. Altarelli and F. Feruglio, Rev. Mod. Phys. 82, 2701 (2010) [arXiv:1002.0211 [hep-ph]]: H. Ishimori, T. Kobayashi, H. Ohki, Y. Shimizu, H. Okada and M. Tanimoto, Prog. Theor. Phys. Suppl. 183, 1 (2010) [arXiv:1003.3552 [hep-th]]; S. F. King and C. Luhn, Rept. Prog. Phys. 76, 056201 (2013) [arXiv:1301.1340 [hep-ph]].

[4] D. Hernandez and A. Y. Smirnov, Phys. Rev. D 86, 053014 (2012) [arXiv:1204.0445 [hep-ph]].

[5] G. Altarelli and D. Meloni, Nucl. Phys. B 809, 158 (2009) [arXiv:0809.1041 [hep-ph]].

[6] D. Meloni, J. Phys. G 37, 055201 (2010) [arXiv:0911.3591 [hep-ph]].

[7] G. L. Fogli, E. Lisi, A. Marrone, D. Montanino, A. Palazzo and A. M. Rotunno, Phys. Rev. D 86, 013012 (2012) [arXiv:1205.5254 [hep-ph]].

[8] A. Donini, D. Meloni and S. Rigolin, Eur. Phys. J. C 45, 73 (2006); A. Donini, E. Fernandez-Martinez, D. Meloni and S. Rigolin, Nucl. Phys. B 743, 41 (2006); D. Meloni and M. Martini, Phys. Lett. B 716, 186 (2012)

[9] D. Meloni, Phys. Lett. B 728, 118 (2014) [arXiv:1308.4578 [hep-ph]].

[10] R. B. Patterson [NOvA Collaboration], arXiv:1209.0716 [hep-ex].

[11] http://www.mpi-hd.mpg.de/personalhomes/globes/index.html; P. Huber, M. Lindner and W. Winter, Comput. Phys. Commun. 167, 195 (2005) [hep-ph/0407333]; P. Huber, J. Kopp, M. Lindner, M. Rolinec and W. Winter, Comput. Phys. Commun. 177, 432 (2007) [hep-ph/0701187].

[12] P. Huber, M. Lindner, T. Schwetz and W. Winter, JHEP 0911, 044 (2009) [arXiv:0907.1896 [hep-ph]].

[13] T. Akiri et al. [LBNE Collaboration], arXiv:1110.6249 [hep-ex].

[14] P. Coloma, P. Huber, J. Kopp and W. Winter, arXiv:1209.5973 [hep-ph].

[15] K. Abe, T. Abe, H. Aihara, Y. Fukuda, Y. Hayato, K. Huang, A. K. Ichikawa and M. Ikeda et al., arXiv:1109.3262 [hep-ex].

[16] A. Donini, D. Meloni and S. Rigolin, JHEP 0406, 011 (2004) [hep-ph/0312072]. 